\title{
Height predict incident non-alcoholic fatty liver disease among general adult population in Tianjin, China, independent of body mass index, waist circumference, waist-to-height ratio, and metabolic syndrome
}

\section{Shubham Kumari}

Tianjin Medical University

\section{Xuena Wang}

Tianjin Medical University

\section{Yunyun Liu}

Tianjin Medical University

\section{Yeqing Gu}

Tianjin Medical University

\section{Yuhan Huang}

Tianjin Medical University

\section{Qing Zhang}

Tianjin Medical University General Hospital

\section{Li Liu}

Tianjin Medical University General Hospital

\section{Ge Meng}

Tianjin Medical University

Hongmei Wu

Tianjin Medical University

Shaomei Sun

Tianjin Medical University General Hospital

\section{Xing Wang}

Tianjin Medical University General Hospital

\section{Ming Zhou}

Tianjin Medical University General Hospital

\section{Qiyu Jia}

Tianjin Medical University General Hospital

\section{Guolin Wang}

Tianjin Medical University General Hospital 


\section{Kun Song}

Tianjin Medical University General Hospital

Kaijun Niu ( $D$ nkj0809@gmail.com )

Tianjin Medical University

\section{Research article}

Keywords: Early life experiences; height; insulin-like growth factor-1; non-alcoholic fatty liver disease.

Posted Date: March 2nd, 2020

DOI: https://doi.org/10.21203/rs.2.17009/v3

License: (c) (1) This work is licensed under a Creative Commons Attribution 4.0 International License. Read Full License

Version of Record: A version of this preprint was published at BMC Public Health on March 24th, 2020. See the published version at https://doi.org/10.1186/s12889-020-08475-1. 


\section{Abstract}

Background : Early-life hormonal and nutritional factors can greatly influence the risk of non-alcoholic fatty liver disease (NAFLD). Adult height is a simple marker for these factors. This study aimed to investigate the association between adult height and NAFLD. Methods : We performed a prospective cohort study of 35,994 participants aged 25 years or over with measured height at baseline. NAFLD was diagnosed by abdominal ultrasound and self-reported history of alcohol intake. Multivariable Cox proportional hazards regression models were conducted to assess the gender-specific association between height and the risk of NAFLD. Results: During a follow-up period of 5.5 years, 6,245 of 35,994 subjects developed NAFLD. The adjusted hazard ratios ( $95 \%$ confidence interval) of NAFLD for increasing quintiles of height were 1.00 (reference), $0.82(0.73,0.92), 0.84(0.73,0.97), 0.72(0.61,0.85)$ and 0.63 $(0.50,0.79)$ ( $P$ for trend $<0.0001)$ in males, and 1.00 (reference), 1.00 (reference), $0.80(0.69,0.91), 0.72$ $(0.61,0.85), 0.60(0.49,0.74)$ and $0.45(0.35,0.59)$ ( $P$ for trend $<0.0001)$ in females, respectively. Conclusions: A higher adult height was associated with lower risk of NAFLD among males and females in Tianjin, China.

\section{Background}

Non-alcoholic fatty liver disease (NAFLD) is defined as the presence of $\geq 5 \%$ of hepatic steatosis, in the absence of secondary causes of hepatic fat accumulation, such as chronic use of medications or significant alcohol intake(1). It is the most common chronic liver disease all over the world and its prevalence is constantly increasing(2). About $25 \%$ of world population was estimated to have NAFLD(3). Unhealthy lifestyles and dietary habits in addition to genetic predisposition have pose increased the prevalence of NAFLD in the Asia Pacific region(4).

Early life experiences, including nutrition and hormone, play an important role in influencing later susceptibility to chronic diseases by epigenetic mechanisms $(5,6)$. Accumulating evidence suggests that hormonal and nutritional experiences in early life may predispose high incidence of type 2 diabetes mellitus (T2DM) and insulin resistance in later life(7). Furthermore, there is sufficient evidence to demonstrate that T2DM, insulin resistance and NAFLD share many important metabolic risk factors and common pathogenetic mechanism(8). Several studies preliminarily suggested that growth hormone (GH) levels and insulin-like growth factor-1 (IGF-1) levels are negatively associated with $\operatorname{NAFLD}$ in adults $(9,10)$, and $\mathrm{GH}$ replacement therapy in GH-deficient patients can alleviate NAFLD and improve liver fibrosis(11). Moreover, suboptimal early life nutrition may increase the susceptibility, age on set, and severity of $\operatorname{NAFLD}(12)$.

Adult height is defined as the tallest height after height velocity had decreased to $1 \mathrm{~cm}$ or less over 6 months and relatively fixed as compared with child or youth height(13). Adult height greatly reflects differences in nutrition and hormone levels in early life $(14,15)$. It is well recognized that GH and IGF-1 are strongly and positively associated with growth in height, and GH therapy in children with short stature caused by several diseases augments adult height(16). Since early hormonal and nutritional experiences 
are major risk factors of final height, it is assumed that adult height may be a potentially useful predictor of incident NAFLD. To date, few studies have focused on the association between adult height and NAFLD.

In the present study, we designed a prospective cohort study to determine whether adult height was associated with the risk of NAFLD among adult population in Tianjin, China.

\section{Methods}

\section{Study design and participants}

Details of the Tianjin Chronic Low-Grade Systemic Inflammation and Health (TCLSIH) Cohort Study have been described elsewhere(17). Briefly, participants were randomly recruited between January 2010 and December 2016 from the general population in Tianjin, China. The inclusion criteria for the TCLSIH cohort study were men and women who were 18 years and older living in Tianjin, China for at least 5 years. Subjects in the present study were sampled by a random process, using a random number generator. Nearly all occupations are covered in this study, and we also included retired individuals living in residential communities. Therefore, the sample population used here is representative of the general adult population in Tianjin, a typical city in north China. All participants received at least 2 health examinations in our study (including liver ultrasound examination, anthropometric measurements, and blood tests) and completed a structured questionnaire survey(18). The questionnaire consisted of the following contents: age, gender, smoking and drinking habits, history of diseases (cardiovascular disease, hypertension, hyperlipidemia, and diabetes), and family history of diseases (cardiovascular disease, hypertension, hyperlipidemia, and diabetes). The reliability and validity of the questionnaire have been assessed, with the Spearman's rank correlation coefficient of 0.67 and 0.58 , separately. Written informed consent was obtained from all participants. The ethical protocol of this study was approved by the Medical Ethics Committee of the Tianjin Medical University with the reference number of TMUhMEC 201430, in accordance with the 1975 Declaration of Helsinki (as revised in 1983).

From 2010 to 2016, a total of 90,536 participants received health examinations. We excluded 170 participants who had missing data on alanine aminotransferase, 4,013 participants had excessive alcohol intake (>140 g/week in males and $>70 \mathrm{~g} /$ week in females), and 28,935 participants who had NAFLD at baseline. Moreover, we excluded 767 participants with other liver diseases (including autoimmune liver diseases, chronic hepatitis B or C, cirrhotic or operation on liver), and those with a history of cardiovascular disease $(n=5,475)$ or cancer $(n=1,039)$, and those aged $<25$ years $(n=3,996)$. Furthermore, participants were also excluded if they were recruited in $2016(n=4,894)$ or were lost in following up $(n=5,253)$. Furthermore, based on the prevalence of NAFLD in Chinese population and on the principle of 10 outcome events per variable (19), the sample size was calculated. Finally, a total of 35,994 participants were available for analysis (follow-up rate: $87 \%$; followed up for 2-5.5 y; mean duration of follow-up (standard deviation): 2.6 (1.6)).

\section{Assessment of height}


Standing height without shoes was measured to the nearest $0.1 \mathrm{~cm}$ using an automatic BMI measuring stadiometer with a precision of $0.1 \mathrm{~cm}$ and a range of 0.9-2.50 m (BSM370, Chungcheongnam-do, Korea). In order to investigate how height level is associated with NAFLD, we divided males and female participants into 5 categories (quintiles) according to height, in $\mathrm{cm}$ (range), as follows: (1) Level 1 (148.5167.7), Level 2 (167.8-171.2), Level 3 (171.3-174.3), Level 4 (174.4-178.1), and Level 5 (178.2-204.1) in males; (2) Level 1 (138.0-156.2), Level 2 (156.3-159.5), Level 3 (159.6-162.1), Level 4 (162.2-165.4), and Level 5 (165.5-184.6) in females.

\section{Diagnosis of NAFLD}

Real-time ultrasonography performed by trained and certified technicians was used to diagnose NAFLD. Participants were considered to have NAFLD if (1) they had a self-reported alcohol intake of $<140 \mathrm{~g} /$ week and $<70 \mathrm{~g} /$ week for males and females, respectively; (2) and at least two of the following abnormal findings of abdominal ultrasound images: diffusely increased liver near field ultrasound echo; increased liver echotexture, compared to the kidneys; vascular blurring and the gradual attenuation of far field ultrasound echo(20). Inter-observer variations for NAFLD status (yes or no) were evaluated in a subsample of 200 participants from the TCLSIH study. The Kappa coefficient was 0.90 , and the total agreement was $96.4 \%$.

\section{Assessment of other variables}

Waist circumference (WC) was measured using a nonelastic plastic anthropometric tape at the level of umbilicus with subjects standing and breathing normally. Waist-to-height ratio was calculated by dividing WC $(\mathrm{cm})$ by the subjects' height $(\mathrm{cm})$. Participants rest for at least 5 minutes in a seated position prior to blood pressure measurements. Blood pressure was measured twice from participants' upper right arms using the TM-2655 device (A\&D Company Ltd, Tokyo, Japan), and the blood pressure value was recorded in average. If the first two results are quite different, additional measurements was carried out until stabilization. The mean of the two closest readings (including the last reading) was calculated to determine the reported BP for each participant. Hypertension was finally assessed and diagnosed by physicians according to the criteria of the JNC 8: hypertension was defined as SBP $\geq 140 \mathrm{mmHg}$ and/or DBP $\geq 90 \mathrm{mmHg}$ or having history of hypertension or using antihypertensive drugs(21). Fasting blood samples for the analysis of biochemical values were collected in siliconized vacuum plastic tubes. Fasting blood glucose was measured by the glucose oxidase method, triglycerides were measured by enzymatic methods, low-density lipoprotein cholesterol was measured by the polyvinyl sulfuric acid precipitation method, high-density lipoprotein cholesterol was measured by the chemical precipitation method, and alanine aminotransferase was measured by International Federation of Clinical Chemists (IFCC) method using reagents from Roche Diagnostics on an automatic biochemistry analyzer (Roche Cobas 8000 modular analyzer, Mannheim, Germany). Diabetes was defined as FBG levels $\geq 7.0 \mathrm{mmol} / \mathrm{L}$ or having history of diabetes. Hyperlipidemia was defined as TC $\geq 5.17 \mathrm{mmol} / \mathrm{L}$ or $\mathrm{TG} \geq 1.7 \mathrm{mmol} / \mathrm{L}$ or $\mathrm{LDL} \geq 3.37 \mathrm{mmol} / \mathrm{L}$ or history of hyperlipidemia. We defined metabolic syndrome (MetS) according to the American Heart Association scientific statements of 2009(22). 
Body weight was measured by an automatic body mass index (BMI) measuring stadiometer (BSM370, Chungcheongnam-do, Korea), accurate to $0.1 \mathrm{~kg}$, with participants wearing only light clothing and no shoes. BMI was calculated as weight $(\mathrm{kg})$ divided by squared height $\left(\mathrm{m}^{2}\right)$. Based on the World Health Organization recommendations for Chinese people, underweight was defined as $\mathrm{BMI}<18.5 \mathrm{~kg} / \mathrm{m}^{2}$, normal weight was defined as $18.5 \mathrm{~kg} / \mathrm{m}^{2} \leq \mathrm{BMI}<23.0 \mathrm{~kg} / \mathrm{m}^{2}$, overweight was defined as $23 \mathrm{~kg} / \mathrm{m}^{2} \leq$ $\mathrm{BMI}<27.5 \mathrm{~kg} / \mathrm{m}^{2}$, and obesity was defined as $\mathrm{BMI} \geq 27.5 \mathrm{~kg} / \mathrm{m}^{2}(23)$. Information on family history of cardiovascular disease, hypertension, hyperlipidemia, and diabetes and lifestyle and health-related habits was assessed at baseline using a structured questionnaire. Smoking status was grouped in three: smoker, ex-smoker or nonsmoker and drinking status was classified as everyday, sometime, ex-drinker or nondrinker by self-reporting.

\section{Statistical analysis}

Baseline characteristics of participants were compared using analysis of variance for continuous variables and logistic regression analysis for categorical variables. Continuous variables were shown as geometric mean (95\% confidence interval $(\mathrm{Cl})$ ), and categorical variables were presented as percentage. Cumulative event rates for incident NAFLD were estimated by Kaplan-Meier survival curves, and equalities were compared with the log-rank test.

We tested the interaction between height and the confounding factors, including age, sex, waist circumference, BMI, smoking status, alcohol drinking status, Mets, and family history of disease (cardiovascular disease, hypertension, hyperlipidemia, and diabetes), separately. The interaction between sex and height was statistically significant $(P<0.0001)$, while the $\mathrm{P}$ values for interaction between height and other confounding factors were $>0.1$. Therefore, we analyzed the association between height and NAFLD stratified by sex. We fitted four Cox proportional hazards regression models to evaluate the association between baseline height and incident NAFLD. The initial model was unadjusted model (crude model). Model 2 was adjusted for age and WC. In model 3, we additionally adjusted for smoking status, alcohol drinking status, Mets, family history of cardiovascular disease, hypertension, hyperlipidemia, and diabetes. In model 4 , we further adjusted for baseline BMI. In model 5, we further adjusted for baseline waist-to-height ratio. Moreover, we adjusted the history of disease (hypertension, diabetes mellitus, and hyperlipidemia) or the subject's blood pressure, fasting blood glucose, triglycerides, low-density lipoprotein cholesterol, and high-density lipoprotein cholesterol to replace the metabolic syndrome in the final multiple-adjusted model, separately. All $P$ values for linear trends were calculated using the median value for each quintile. All statistical analyses were performed using SAS version 9.4 (SAS Institute, Inc.). Two-tailed $P<0.05$ was considered as statistically significant.

\section{Results}

In this study, $41.3 \%$ of participants were males. Mean age was $42.2 \pm 12.9$ years for males and $39.2 \pm 11.3$ years for females. In males, the mean BMI was $24.0 \pm 2.9 \mathrm{~kg} / \mathrm{m}^{2}$, corresponding to $41.8 \%$ with BMI category of overweight, and $7.9 \%$ with BMI category of obesity. In females, the mean BMI was $22.1 \pm 2.9$ 
$\mathrm{kg} / \mathrm{m}^{2}$, corresponding to $20.3 \%$ with BMI category of overweight, and $3.4 \%$ with BMI category of obesity. During the 5.5 years of the study period (2010-2016), 6,245 of 35,994 individuals (17.4\%) developed NAFLD. Incidence of NAFLD was 65.8 per 1,000 person-years. The cumulative incidence of NAFLD was significantly lower in subjects in higher quintiles of baseline height compared with those with the lowest quintile (Fig. 1, $P<0.0001$ by log-rank test). Subgroup analysis showed NAFLD incidence of 110.1, 104.4, 113.2, 108.7 and 110.2 per 1000 person-years in males and 48.4, 43.1, 40.7, 35.9 and 31.7 per 1,000 person-years in females, for 1 to 5 height quintiles, respectively.

Characteristics of participants relative to incident NAFLD status for follow-up analysis are presented in Table 1. The mean age $(95 \% \mathrm{Cl})$ in non-NAFLD and NAFLD participants was $38.3(38.2,38.4)$ years and $41.4(41.1,41.7)$ years, respectively. Compared with participants who did not develop NAFLD, participants who developed NAFLD had older age, lower high-density lipoprotein cholesterol, but higher BMI, WC, total cholesterol, low-density lipoprotein cholesterol, triglycerides, fasting blood glucose, systolic blood pressure, diastolic blood pressure, and alanine aminotransferase (all $P<0.0001$ ). Participants who developed NAFLD tended to be male, smoker, ex-smoker, everyday drinker, sometimes drinker, while those who did not develop NAFLD tended to be non-smoker and non-drinker (all $P<0.0001$ ). In addition, a higher proportion of participants who developed NAFLD had MetS and a family history of cardiovascular disease, hypertension and diabetes (all $P<0.0001$ ).

Table 2 and Table 3 show the crude and adjusted associations between quintiles of height and NAFLD in male and female participants, respectively. In the third multivariate model which didn't adjust for BMI , the adjusted HRs $(95 \% \mathrm{Cl})$ for NAFLD across height quintiles were 1.00 (reference), $0.86(0.78,0.95), 0.90$ $(0.81,0.99), 0.77(0.70,0.85)$ and $0.71(0.64,0.78)$ in males and 1.00 (reference), $0.92(0.82,1.04), 0.85$ $(0.75,0.97), 0.78(0.68,0.89)$ and $0.65(0.57,0.74)$ in females (both $P$ for trend $<0.0001)$. Similarly, in the fourth multivariate model which adjusted $\mathrm{BMI}$, the adjusted HRs $(95 \% \mathrm{Cl})$ for NAFLD across height quintiles were 1.00 (reference), $0.90(0.81,0.99), 0.97(0.87,1.07), 0.86(0.78,0.96)$ and $0.84(0.75,0.94)$ in males $(P$ for trend $<0.01)$ and 1.00 (reference), $0.97(0.86,1.09), 0.98(0.86,1.11), 0.93(0.81,1.06)$ and $0.84(0.73,0.96)$ in females $(P$ for trend $=0.02)$. In the final multivariate model which adjusted waist-toheight ratio, the adjusted HRs $(95 \% \mathrm{Cl})$ for NAFLD across height quintiles were 1.00 (reference), 0.82 $(0.73,0.92), 0.84(0.73,0.97), 0.72(0.61,0.85)$ and $0.63(0.50,0.79)$ in males $(P$ for trend $<0.0001)$ and 1.00 (reference), $0.80(0.69,0.91), 0.72(0.61,0.85), 0.60(0.49,0.74)$ and $0.45(0.35,0.59)$ in females $(P$ for trend $<0.0001)$.

When including the history of disease (hypertension, diabetes mellitus, and hyperlipidemia) in the final multiple-adjusted model, the adjusted HRs $(95 \% \mathrm{Cl})$ for NAFLD across height quintiles were 1.00 (reference), $0.85(0.75,0.96), 0.87(0.75,1.01), 0.76(0.63,0.91)$ and $0.65(0.51,0.83)$ in males $(P$ for trend $<0.001)$ and 1.00 (reference), $0.75(0.65,0.87), 0.72(0.6,0.87), 0.6(0.48,0.75)$ and $0.44(0.33,0.6)$ in females ( $P$ for trend $<0.0001$ ). When including the subject's blood pressure, fasting blood glucose, triglycerides, low-density lipoprotein cholesterol and high-density lipoprotein cholesterol in the final multiple-adjusted model, the adjusted HRs $(95 \% \mathrm{Cl})$ for NAFLD across height quintiles were 1.00 (reference), $0.84(0.74,0.96), 0.87(0.75,1.02), 0.76(0.63,0.92)$ and $0.64(0.49,0.82)$ in males ( $P$ for trend 
$<0.001)$ and 1.00 (reference), $0.79(0.67,0.92), 0.78(0.64,0.95), 0.63(0.49,0.79)$ and $0.48(0.36,0.66)$ in females ( $P$ for trend $<0.0001)$.

\section{Discussion}

In this large-scale prospective cohort study, we found that higher level of adult height was inversely associated with the risk of NAFLD among males and females in Tianjin, China. The inverse association remained even after controlling for potential confounding factors. To our knowledge, this is the first study to investigate the association between adult height and NAFLD.

We adjusted for multiple potentially confounding factors in our analysis. This study indicated that numerous factors (such as age, BMI, WC, smoking status, metabolic syndrome and family history of some diseases) correlated positively with NAFLD. We used crude model (model 1) first and results showed negative association between height and NAFLD in both males and females. It is well-recognized that NAFLD and height are related to age and $W C(24,25)$, so we adjusted for these two variables in model 2. Adjustment for age and WC made the associations in males more obvious compared with model 1 ; however, in females, this adjustment didn't significantly influence the associations in model 1, leading us to conclude that age and WC are major confounding factors in males but not in females. Since NAFLD was associated with WC, smoking status, drinking status, metabolic syndrome, family history of cardiovascular disease, hypertension, hyperlipidemia and diabetes(26, 27), we additionally adjusted for these variables in model 3. After adjustments for these factors, the associations didn't change significantly in both males and females, implying these factors may not confound the association between height and NAFLD. In model 4, the present study adjusted BMI and variables in model 3 to confirm the role of BMI in association between height and NAFLD. This adjustment made the association less obvious in both males and females, suggesting that BMI play an important role in association between height and NAFLD. In addition, because waist-to-height ratio has been reported to have a strong association with NAFLD (28), we further adjusted waist-to-height ratio in model 5 . The enhanced associations indicated the confounding effect of waist-to-height ratio.

To date, no studies have investigated the association between height and NAFLD. Several studies investigated the association between height and T2DM. Reports about the risk of T2DM and height have produced conflicting results $(29,30)$. A meta-analysis showed negative association between height and risk of T2DM in woman only(31), whereas a cohort study of Finnish men showed adult height is associated with decreased risk of T2DM (1). Since T2DM and NAFLD both result from metabolic dysregulation, these results are, to some extent, consistent with our novel findings that shorter people were associated with higher incidence of NAFLD. Compared to previous cohort studies using Chinese adults with overall incidence of NAFLD ranging from $15.2 \%$ to $24.8 \%(32)$, the overall incidence of NAFLD in our study is $17.4 \%$, consistent with previous studies.

Although studies of the association between height and NAFLD were sparse, other clinical markers of body composition have been found to be associated with the risk of NAFLD. A prospective study found 
that the risk of NAFLD increased linearly with increasing BMI (33). A retrospective cohort study reported that BMI was a useful predictive factor for NAFLD onset (34). Another study also implied that a greater BMI increase in midlife predicted a greater risk of developing NAFLD (35). In addition, WC was reported to predict NAFLD with a similar performance with fatty liver index (36). A population-based study also pointed out that body roundness index and waist-to-height ratio were strongly associated with NAFLD (28). Results in our current study indicated a substantial inverse association between height and NAFLD risk, regardless of BMI, waist circumference and waist-to-height ratio. Although height is generally nonmodifiable, increasing awareness of its potential effects may contribute to the formulation of more accurate risk prediction models and may help subjects to prevent the onset of NAFLD and the development of further complications by changing their other behaviors.

The mechanisms of association between adult height and NAFLD may involve epigenetic changes induced by early life adversity. Interacting with epigenetic mechanisms, hormonal and nutritional conditions in early life influence both attained height and later susceptibility to NAFLD. Although height is shown to be associated with NAFLD, it may be an indicator of risk that likely reflects the hormonal conditions in early life, which are thought to affect health during adulthood (37). It is well-known that growth hormone (GH) and insulin-like growth factor-I (IGF-I) play essential roles in linear growth (38). Recently, growing body of evidence has revealed the essential roles of GH and IGF-I in liver metabolism (39). GH promotes fat metabolism and reduces visceral fat(40), which was closely associated with the progression of NAFLD. IGF-I induces cellular senescence, inactivates hepatic stellate cells, and thus ameliorating fibrosis (41). Therefore, hormonal conditions in early life may be a potential explanation of the link between greater height and lower NAFLD risk. In addition, data from animal models have indicated that nutritional perturbation of epigenetic regulation is a likely link between prenatal and early postnatal nutrition and health status in later life(42). A study in mice showed that exposure to prenatal and post-weaning western-style diet predisposed male mouse offspring to the development of NAFLD in adulthood and induced alterations in DNA methylation in key metabolic genes(43). Biological links, although plausible, remain speculative. Further research is warranted to validate the hypotheses.

The present study has several limitations. Firstly, NAFLD was diagnosed by abdominal ultrasound rather than liver biopsy, which is the gold standard for diagnosis of NAFLD. Ultrasonographic examination is not able to quantify the liver fat or differentiate different stages of NAFLD. Thus, the associations between height and progressive liver disease could not be evaluated in this study. However, abdominal ultrasound is not invasive and widely used in large-scale population-based studies. Moreover, this noninvasive method has a sensitivity of $89 \%$ and a specificity of $93 \%(44)$. Second, although we adjusted for a considerable number of potential confounding factors in the present study, residual confounding cannot be excluded. Third, liver mass has been reported to be predicted by the square of height (45). However, the present study did not measure liver mass, the effect of which on the association between height and the risk of NAFLD cannot be ruled out. Thus, more studies are required to confirm the role of liver mass. Fourth, even though this study population was comprised of general adults in Tianjin, China, the results may be applicable to other similar populations. Fifth, we used the waist circumference measured at the 
umbilicus in this study. However, waist circumference measured at the iliac crest was reported to be more suitable for use in clinical practice.

\section{Conclusions}

Adult height was negatively associated with the incidence of NAFLD among males and females in Tianjin, China, independent of BMI, WC, waist-to-height ratio, and MetS. The present results indicate that adult height may be a useful predictor for NAFLD to identify high-risk populations and prevent NAFLD at an early age. Such early identification may allow individuals to change their lifestyles to prevent the onset of NAFLD and the development of further complications, which have clinical and public health implications for present and future generations. Future studies are needed to elucidate the mechanism of association between adult height and the risk of NAFLD.

\section{List Of Abbreviations}

NAFLD Non-alcoholic fatty liver disease

T2DM Type 2 diabetes mellitus

GH Growth hormone

IGF-1 Insulin-like growth factor-1

WC Waist circumference

TCLSIH Tianjin Chronic Low-Grade Systemic Inflammation and Health

MetS Metabolic syndrome

IFCC International Federation of Clinical Chemists

BMI Body mass index

$\mathrm{Cl} \quad$ Confidence interval

HR Hazard ratio

\section{Declarations}

\section{Ethics approval and consent to participate}

The ethical protocol of this study was approved by the Medical Ethics Committee of the Tianjin Medical University with the reference number of TMUhMEC 201430, in accordance with the 1975 Declaration of 
Helsinki (as revised in 1983). Each participant gave written informed consent prior to participation in the study.

\section{Consent for publication}

Not applicable

\section{Availability of data and materials}

The datasets generated and analysed during the current study are not publicly available due [public availability would compromise participant privacy] but are available from the corresponding author on reasonable request.

\section{Competing interests}

The authors declare that they have no competing interests.

\section{Funding}

This work was supported by grants from the National Natural Science Foundation of China (No. $81673166,81372118,81372467$ and 81302422$)$, China. The funder of the study had no role in study design, data collection, data analysis, data interpretation, or writing of the report.

\section{Authors' contributions}

Conception and design of the study: S.K., X.W., and K.N. Data collection and analysis: S.K., X.W., Y.G., Y.H., Q.Z., L.L., G.M., H.W., S.S., X.W., M.Z., Q.J., G.W., K.S., and K.N. Interpretation of data: S.K., X.W., Y.L., and K.N. Drafting the manuscript: S.K., X.W., Y.L., and K.N. All authors read and approved the final manuscript.

\section{Acknowledgements}

We gratefully acknowledge all of the people who participated in the study and Tianjin Medical University General Hospital-Health Management Centre for creating the possibility to perform the study.

\section{References}

1. Anstee QM, McPherson S, Day CP. How big a problem is non-alcoholic fatty liver disease? BMJ. 2011;343:d3897.

2. Woo Baidal JA, Lavine JE. The intersection of nonalcoholic fatty liver disease and obesity. Science translational medicine. 2016;8(323):323rv1.

3. Younossi ZM, Koenig AB, Abdelatif D, Fazel Y, Henry L, Wymer M. Global epidemiology of nonalcoholic fatty liver disease-Meta-analytic assessment of prevalence, incidence, and outcomes. Hepatology (Baltimore, Md). 2016;64(1):73-84. 
4. Duseja A, Chalasani N. Epidemiology and risk factors of nonalcoholic fatty liver disease (NAFLD). Hepatology international. 2013;7 Suppl 2:755-64.

5. Waterland RA, Jirtle RL. Early nutrition, epigenetic changes at transposons and imprinted genes, and enhanced susceptibility to adult chronic diseases. Nutrition (Burbank, Los Angeles County, Calif). 2004;20(1):63-8.

6. Wang G, Walker SO, Hong X, Bartell TR, Wang X. Epigenetics and early life origins of chronic noncommunicable diseases. The Journal of adolescent health : official publication of the Society for Adolescent Medicine. 2013;52(2 Suppl 2):S14-21.

7. Jiang $X$, Ma H, Wang Y, Liu Y. Early life factors and type 2 diabetes mellitus. Journal of diabetes research. 2013;2013:485082.

8. Bugianesi E, Moscatiello S, Ciaravella MF, Marchesini G. Insulin resistance in nonalcoholic fatty liver disease. Current pharmaceutical design. 2010;16(17):1941-51.

9. Xu L, Xu C, Yu C, Miao M, Zhang X, Zhu Z, et al. Association between serum growth hormone levels and nonalcoholic fatty liver disease: a cross-sectional study. PloS one. 2012;7(8):e44136.

10. Arturi F, Succurro E, Procopio C, Pedace E, Mannino GC, Lugara M, et al. Nonalcoholic fatty liver disease is associated with low circulating levels of insulin-like growth factor-I. The Journal of clinical endocrinology and metabolism. 2011;96(10):E1640-4.

11. Nishizawa H, Iguchi G, Murawaki A, Fukuoka H, Hayashi Y, Kaji H, et al. Nonalcoholic fatty liver disease in adult hypopituitary patients with GH deficiency and the impact of GH replacement therapy. European journal of endocrinology. 2012;167(1):67-74.

12. Li M, Reynolds CM, Segovia SA, Gray C, Vickers MH. Developmental Programming of Nonalcoholic Fatty Liver Disease: The Effect of Early Life Nutrition on Susceptibility and Disease Severity in Later Life. BioMed research international. 2015;2015:437107.

13. Taback SP, Collu R, Deal CL, Guyda HJ, Salisbury S, Dean HJ, et al. Does growth-hormone supplementation affect adult height in Turner's syndrome? Lancet (London, England). 1996;348(9019):25-7.

14. Silventoinen K. Determinants of variation in adult body height. Journal of biosocial science. 2003;35(2):263-85.

15. Deodati A, Cianfarani S. Impact of growth hormone therapy on adult height of children with idiopathic short stature: systematic review. Bmj. 2011;342:c7157.

16. Lin-Su K, Harbison MD, Lekarev O, Vogiatzi MG, New MI. Final adult height in children with congenital adrenal hyperplasia treated with growth hormone. The Journal of clinical endocrinology and metabolism. 2011;96(6):1710-7.

17. Gu Y, Li H, Bao X, Zhang Q, Liu L, Meng G, et al. The Relationship Between Thyroid Function and the Prevalence of Type 2 Diabetes Mellitus in Euthyroid Subjects. The Journal of clinical endocrinology and metabolism. 2017;102(2):434-42.

18. Gu Y, Chi VTQ, Zhang Q, Liu L, Meng G, Wu H, et al. Low-Normal Thyroid Function Predicts Incident Anemia in the General Population With Euthyroid Status. J Clin Endocrinol Metab. 
2019;104(11):5693-702.

19. Vittinghoff $E$, McCulloch $C E$. Relaxing the rule of ten events per variable in logistic and Cox regression. American journal of epidemiology. 2007;165(6):710-8.

20. Gao X, Fan JG. Diagnosis and management of non-alcoholic fatty liver disease and related metabolic disorders: consensus statement from the Study Group of Liver and Metabolism, Chinese Society of Endocrinology. Journal of diabetes. 2013;5(4):406-15.

21. James PA, Oparil S, Carter BL, Cushman WC, Dennison-Himmelfarb C, Handler J, et al. 2014 evidence-based guideline for the management of high blood pressure in adults: report from the panel members appointed to the Eighth Joint National Committee (JNC 8). Jama. 2014;311(5):507-20.

22. Alberti KG, Eckel RH, Grundy SM, Zimmet PZ, Cleeman JI, Donato KA, et al. Harmonizing the metabolic syndrome: a joint interim statement of the International Diabetes Federation Task Force on Epidemiology and Prevention; National Heart, Lung, and Blood Institute; American Heart Association; World Heart Federation; International Atherosclerosis Society; and International Association for the Study of Obesity. Circulation. 2009;120(16):1640-5.

23. Appropriate body-mass index for Asian populations and its implications for policy and intervention strategies. Lancet (London, England). 2004;363(9403):157-63.

24. Frith J, Day CP, Henderson E, Burt AD, Newton JL. Non-alcoholic fatty liver disease in older people. Gerontology. 2009;55(6):607-13.

25. Foghsgaard S, Andreasen C, Vedtofte L, Andersen ES, Bahne E, Strandberg C, et al. Nonalcoholic Fatty Liver Disease Is Prevalent in Women With Prior Gestational Diabetes Mellitus and Independently Associated With Insulin Resistance and Waist Circumference. Diabetes care. 2017;40(1):109-16.

26. Loomba R, Abraham M, Unalp A, Wilson L, Lavine J, Doo E, et al. Association between diabetes, family history of diabetes, and risk of nonalcoholic steatohepatitis and fibrosis. Hepatology (Baltimore, Md). 2012;56(3):943-51.

27. Ghamar-Chehreh ME, Khedmat $H$, Amini M, Taheri S. Predictive value of having positive family history of cardiovascular disorders, diabetes mellitus, dyslipidemia, and hypertension in nonalcoholic fatty liver disease patients. Acta medica Iranica. 2013;51(5):307-13.

28. Motamed N, Rabiee B, Hemasi GR, Ajdarkosh H, Khonsari MR, Maadi M, et al. Body Roundness Index and Waist-to-Height Ratio are Strongly Associated With Non-Alcoholic Fatty Liver Disease: A Population-Based Study. Hepatitis monthly. 2016;16(9):e39575.

29. Lorenzo C, Williams K, Stern MP, Haffner SM. Height, ethnicity, and the incidence of diabetes: the San Antonio Heart Study. Metabolism: clinical and experimental. 2009;58(11):1530-5.

30. Janghorbani M, Momeni F, Dehghani M. Hip circumference, height and risk of type 2 diabetes: systematic review and meta-analysis. Obesity reviews : an official journal of the International Association for the Study of Obesity. 2012;13(12):1172-81.

31. Vangipurapu J, Stancakova A, Jauhiainen R, Kuusisto J, Laakso M. Short Adult Stature Predicts Impaired beta-Cell Function, Insulin Resistance, Glycemia, and Type 2 Diabetes in Finnish Men. J Clin 
Endocrinol Metab. 2017;102(2):443-50.

32. Yang C, Yang S, Xu W, Zhang J, Fu W, Feng C. Association between the hyperuricemia and nonalcoholic fatty liver disease risk in a Chinese population: A retrospective cohort study. PloS one. 2017;12(5):e0177249.

33. Loomis AK, Kabadi S, Preiss D, Hyde C, Bonato V, St Louis M, et al. Body Mass Index and Risk of Nonalcoholic Fatty Liver Disease: Two Electronic Health Record Prospective Studies. J Clin Endocrinol Metab. 2016;101(3):945-52.

34. Miyake T, Kumagi T, Hirooka M, Furukawa S, Koizumi M, Tokumoto Y, et al. Body mass index is the most useful predictive factor for the onset of nonalcoholic fatty liver disease: a community-based retrospective longitudinal cohort study. J Gastroenterol. 2013;48(3):413-22.

35. VanWagner LB, Khan SS, Ning H, Siddique J, Lewis CE, Carr JJ, et al. Body mass index trajectories in young adulthood predict non-alcoholic fatty liver disease in middle age: The CARDIA cohort study. Liver international : official journal of the International Association for the Study of the Liver. 2018;38(4):706-14.

36. Motamed N, Sohrabi M, Ajdarkosh H, Hemmasi G, Maadi M, Sayeedian FS, et al. Fatty liver index vs waist circumference for predicting non-alcoholic fatty liver disease. World journal of gastroenterology. 2016;22(10):3023-30.

37. Gunnell D, Whitley E, Upton MN, McConnachie A, Smith GD, Watt GC. Associations of height, leg length, and lung function with cardiovascular risk factors in the Midspan Family Study. Journal of epidemiology and community health. 2003;57(2):141-6.

38. Ranke MB, Wit JM. Growth hormone - past, present and future. Nat Rev Endocrinol. 2018;14(5):285300.

39. Takahashi Y. Essential roles of growth hormone (GH) and insulin-like growth factor-I (IGF-I) in the liver. Endocrine journal. 2012;59(11):955-62.

40. <2 Scaling of human body composition to stature.pdf>.

41. Nishizawa H, Iguchi G, Fukuoka H, Takahashi M, Suda K, Bando H, et al. IGF-I induces senescence of hepatic stellate cells and limits fibrosis in a p53-dependent manner. Scientific reports. 2016;6:34605.

42. Holland ML, Lowe R, Caton PW, Gemma C, Carbajosa G, Danson AF, et al. Early-life nutrition modulates the epigenetic state of specific rDNA genetic variants in mice. Science (New York, NY). 2016;353(6298):495-8.

43. Pruis MG, Lendvai A, Bloks VW, Zwier MV, Baller JF, de Bruin A, et al. Maternal western diet primes non-alcoholic fatty liver disease in adult mouse offspring. Acta physiologica (Oxford, England). 2014;210(1):215-27.

44. Saadeh S, Younossi ZM, Remer EM, Gramlich T, Ong JP, Hurley M, et al. The utility of radiological imaging in nonalcoholic fatty liver disease. Gastroenterology. 2002;123(3):745-50.

45. Heymsfield SB, Gallagher D, Mayer L, Beetsch J, Pietrobelli A. Scaling of human body composition to stature: new insights into body mass index. The American journal of clinical nutrition. 2007;86(1):8291. 


\section{Tables}

Page 15/22 
Table 1. Baseline characteristics of participants by incident NAFLD status ${ }^{a}$.

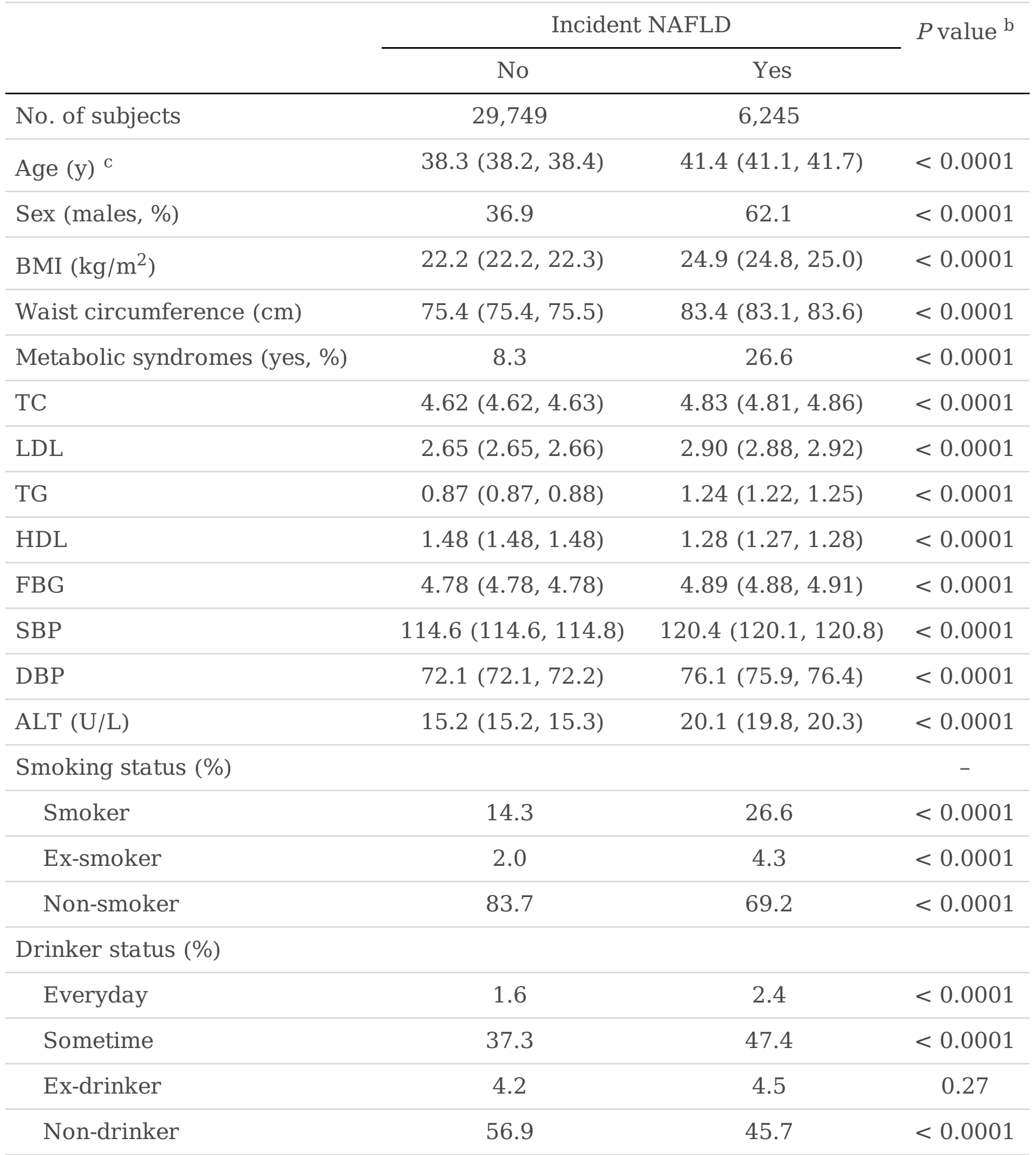

Family history of diseases (\%) 


\begin{tabular}{lccc}
\hline CVD & 28.1 & 34.8 & $<0.0001$ \\
\hline Hypertension & 48.0 & 55.3 & $<0.0001$ \\
\hline Hyperlipidemia & 0.5 & 0.6 & 0.055 \\
\hline Diabetes & 20.7 & 26.0 & $<0.0001$ \\
\hline
\end{tabular}

a NAFLD, non-alcoholic fatty liver disease; BMI, body mass index; MS, metabolic syndromes; TC, total cholesterol; LDL, low-density lipoprotein cholesterol; TG, triglycerides; HDL, high-density lipoprotein cholesterol; FBG, fasting blood glucose; SBP, systolic blood pressure; DBP, diastolic blood pressure; ALT, alanine aminotransferase; CVD, cardiovascular disease.

$\mathrm{b}$ Analysis of variance or logistic regression analysis.

c Geometric mean (95\% confidence interval) (all such values). 
Table 2. Cohort analysis: adjusted associations of height quintiles with NAFLD ${ }^{a}$ in males.

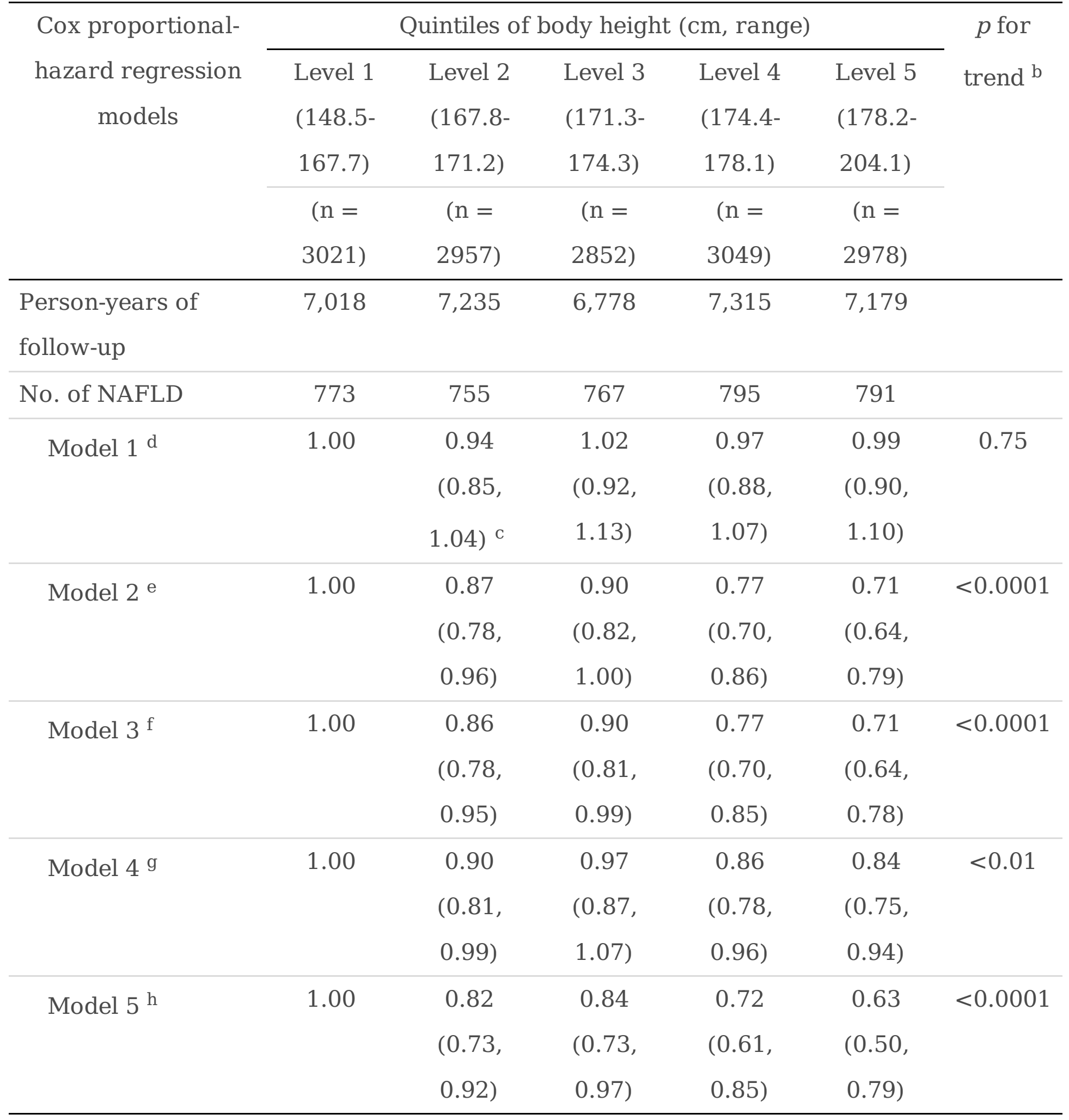
a NAFLD, non-alcoholic fatty liver disease.
b Analysis by Cox proportional hazards model.
C Adjusted hazard ratios (95\% confidence interval) (all such values). 
d Crude

e Adjusted for age and waist circumference.

f Adjusted for age, waist circumference, smoking status, drinking status, metabolic syndrome, and family history of cardiovascular disease, hypertension, hyperlipidemia, and diabetes.

g Adjusted for age, body mass index, waist circumference, smoking status, drinking status, metabolic syndrome, and family history of cardiovascular disease, hypertension, hyperlipidemia, and diabetes.

$\mathrm{h}$ Adjusted for age, body mass index, waist-to-height ratio, waist circumference, smoking status, drinking status, metabolic syndrome, and family history of cardiovascular disease, hypertension, hyperlipidemia, and diabetes. 
Table 3. Cohort analysis: adjusted associations of height quintiles with NAFLD ${ }^{a}$ in females.

\begin{tabular}{|c|c|c|c|c|c|c|}
\hline \multirow{6}{*}{$\begin{array}{l}\text { Cox proportional- } \\
\text { hazard regression } \\
\text { models }\end{array}$} & \multicolumn{5}{|c|}{ Quintiles of body height (cm, range) } & \multirow{6}{*}{$\begin{array}{l}p \text { for } \\
\text { trend }{ }^{b}\end{array}$} \\
\hline & Level 1 & Level 2 & Level 3 & Level 4 & Level 5 & \\
\hline & $(138.0-$ & (156.3- & $(159.6-$ & $(162.2-$ & $(165.5-$ & \\
\hline & 156.2) & 159.5) & 162.1) & 165.4) & 184.6) & \\
\hline & $(\mathrm{n}=$ & $(\mathrm{n}=$ & $(\mathrm{n}=$ & $(\mathrm{n}=$ & $(\mathrm{n}=$ & \\
\hline & 4223) & 4442) & 3947) & 4145) & 4370) & \\
\hline \multirow{2}{*}{$\begin{array}{l}\text { Person-years of } \\
\text { follow-up }\end{array}$} & 11,318 & 12,593 & 11,153 & 11,576 & 12,736 & \\
\hline & & & & & & \\
\hline No. of NAFLD & 548 & 543 & 454 & 416 & 404 & \\
\hline \multirow[t]{3}{*}{ Model 1 d } & 1.00 & 0.88 & 0.83 & 0.73 & 0.64 & $<0.0001$ \\
\hline & & $(0.78$ & $(0.73$ & $(0.64$ & (0.57, & \\
\hline & & $0.99)^{\mathrm{c}}$ & $0.94)$ & 0.83) & 0.73) & \\
\hline \multirow[t]{3}{*}{ Model $2 \mathrm{e}$} & 1.00 & 0.91 & 0.85 & 0.75 & 0.62 & $<0.0001$ \\
\hline & & (0.81, & (0.75, & (0.66, & (0.55, & \\
\hline & & 1.02) & 0.96) & 0.85) & $0.71)$ & \\
\hline \multirow[t]{3}{*}{ Model 3 f } & 1.00 & 0.92 & 0.85 & 0.78 & 0.65 & $<0.0001$ \\
\hline & & $(0.82$, & (0.75, & (0.68, & (0.57, & \\
\hline & & 1.04) & 0.97) & 0.89) & $0.74)$ & \\
\hline \multirow[t]{3}{*}{ Model $4 \mathrm{~g}$} & 1.00 & 0.97 & 0.98 & 0.93 & 0.84 & 0.02 \\
\hline & & $(0.86$ & $(0.86$ & (0.81, & $(0.73$ & \\
\hline & & 1.09) & 1.11) & 1.06) & 0.96) & \\
\hline \multirow[t]{3}{*}{ Model $5^{h}$} & 1.00 & 0.80 & 0.72 & 0.60 & 0.45 & $<0.0001$ \\
\hline & & (0.69, & (0.61, & $(0.49$ & (0.35, & \\
\hline & & 0.91) & 0.85) & $0.74)$ & 0.59) & \\
\hline
\end{tabular}

a NAFLD, non-alcoholic fatty liver disease.

b Analysis by Cox proportional hazards model.

C Adjusted hazard ratios (95\% confidence interval) (all such values). 
d Crude

e Adjusted for age and waist circumference.

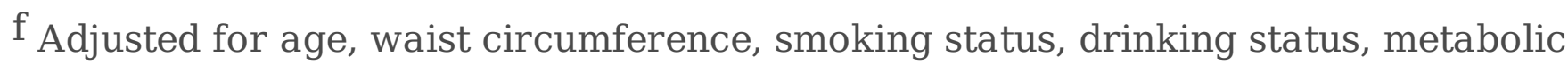
syndrome, and family history of cardiovascular disease, hypertension, hyperlipidemia, and diabetes.

g Adjusted for age, body mass index, waist circumference, smoking status, drinking status, metabolic syndrome, and family history of cardiovascular disease, hypertension, hyperlipidemia, and diabetes.

$\mathrm{h}$ Adjusted for age, body mass index, waist-to-height ratio, waist circumference, smoking status, drinking status, metabolic syndrome, and family history of cardiovascular disease, hypertension, hyperlipidemia, and diabetes.

\section{Figures}

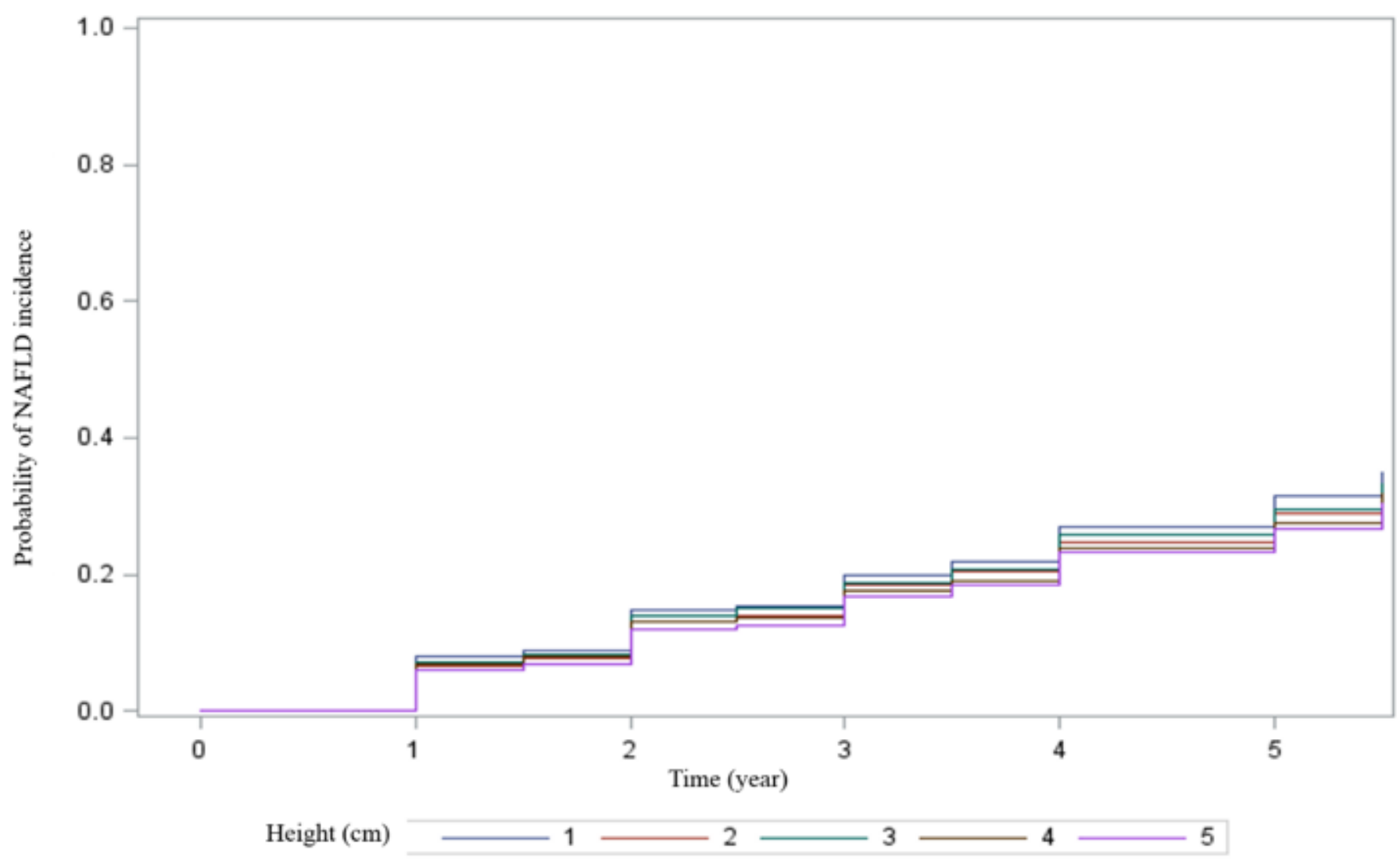

Figure 1 
Five-year NAFLD incidence according to baseline height quintiles. 